\title{
DESORPTION OF PHOSPHATE FROM GOETHITE
}

\author{
L. MADRID* and A. M. POSNER \\ (Department of Soil Science and Plant Nutrition, University of Westem Australia, Nedlands, \\ W.A. 6009, Australia.)
}

\begin{abstract}
Summary
The reversibility of the adsorption of phosphate on goethite was measured by diluting suspensions of goethite on which phosphate was adsorbed with large volumes of phospluate-free solution at the same ionic strength. The effect on the reversibility of various adsorption and desorption conditions was studied, particularly $\mathrm{pH}$, temperature, ionic strength and time of reaction.

The apparent irreversibility of the adsorption reaction seems to be due to a slow adsorption reaction of part of the phosphate after a very rapid initial adsorption, with a similar behaviour when the phosphate is desorbed. When the total adsorption plus desorption time is long enough, both adsorption and desorption points tend to lie on a single curve which corresponds to the iso therm calculated according to the Stern doublelayer theory.
\end{abstract}

\section{Introduction}

DESPITE the considerable volume of work done by many authors on adsorption of phosphate by mineral surfaces, comparatively little progress has been made in studies of desorption. There is evidence that when solids on which phosphate is adsorbed are washed at a constant electrolyte concentration and $\mathrm{pH}$, the desorption points are located above the adsorption isotherms (Muljadi et al., 1966; Kafkafi et al., 1967; Hingston et al., 1974) demonstrating the presence of hysteresis, the origins of which are not clear. Atkinson et al. (1972) found that most of the phosphate adsorbed on goethite was isotopically exchangeable, which means that it should be in equilibrium with the solution, and Neoh (1975) found a high proportion of the phosphate remaining on goethite after desorption to be isotopically exchangeable. These facts suggest that the hysteresis is due to the very slow desorption of the so-called 'non-desorbable' phosphate.

Hingston et al. (1974) believed that the lack of reversibility of the isotherms could be related to the kinetics of adsorption and desorption. Later, other authors, e.g. Barrow and Shaw (1975), Munns and Fox (1976), Ryden and Syers (1977) and White and Taylor (1977), have also suggested that the apparent irreversibility of adsorption isotherms can be due to slow equilibration during adsorption. They found that there is even an apparent re-adsorption during the desorption step, attributed to a very slow adsorption reaction for part of the phosphate. Ry den and Syers (1977) believe that the initially desorbable phosphate (which they call 'region III' or 'more-physically sorbed') undergoes a shift to 'chemisorbed' forms ('regions I and II' of their isotherms), which can only be desorbed with an increase in the $\mathrm{pH}$

\footnotetext{
* Permanent Address: Centro de Edafologia y Biologia Aplicada del Cuarto, C.S.I.C. Apartado 1052, Sevilla, Spain.
}

Journal of Soil Science, $1979,30,697-707$ 
of the desorbing solution. A similar idea had been previously suggested by Muljadi et al. (1966).

In the experiments described here, the aim was to investigate the reversibility of the adsorption of phosphate on goethite as influenced by the conditions of adsorption and desorption.

\section{Experimental}

The preparation of the goethite has been described elsewhere (Hingston et al., 1972). Its specific surface area was $84 \mathrm{~m}^{2} \mathrm{~g}^{-1}$ and its pzc was at $\mathrm{pH} 8$.

\section{Adsorption experiments}

Six $\mathrm{ml}$ of a well-stirred suspension of goethite in water $\left(19.5 \mathrm{mg} \mathrm{ml}^{-1}\right)$ was added to polythene bottles containing $50 \mathrm{ml}$ of $\mathrm{NaCl}$ solutions of varying ionic strengths, $\mathrm{pH}$ and temperatures, with known concentrations of ${ }^{31} \mathrm{P}+{ }^{32} \mathrm{P}$. After allowing a given time of adsorption, samples of these suspensions were filtered through $0.2 \mu \mathrm{m}$ Millipore filters, and ${ }^{32} \mathrm{P}$ was determined by Cherenkov counting. Sufficient solution was passed through the filter before collecting the filtrate for counting to avoid losses due to adsorption. The final phosphate concentration and amount adsorbed were calculated by means of the ${ }^{31} \mathrm{P}:{ }^{32} \mathrm{P}$ ratio of the original solution used. The solid : solution ratio was $0.0021 \mathrm{~g}_{\text {goethite }} \mathrm{ml}^{-1}$.

\section{Desorption experiments}

After each adsorption period, samples of the adsorption suspensions were pipetted into polythene bottles containing large known volumes of solutions, at various conditions of $\mathrm{pH}$ and temperature, but always at the same ionic strength as in the adsorption experiment. The resulting 'desorption suspensions' had a solid : solution ratio from 17 to 3000 times less than in the adsorption experiments.

From these 'desorption suspensions' samples were taken at various times and filtered through Millipore filters. The phosphate concentration and the amount remaining on the oxide were determined as described for the adsorption experiments.

All suspensions were shaken in an orbital shaker at 180 r.p.m., except those in which the reaction time was less than $5 \mathrm{~min}$, which were shaken by hand.

In some experiments, $\mathrm{LiCl}$ was used instead of $\mathrm{NaCl}$ as indifferent electrolyte. The experiments at very high $\mathrm{pH}$ were made in polythene bottles wrapped with aluminium foil to reduce contamination with $\mathrm{CO}_{2}$. For reasons that will be explained later, polycarbonate bottles were sometimes used instead of polythene. To find out whether the technique had an influence upon the amount adsorbed or desorbed, some experiments were made by the adsorption-desorption technique used by Hingston et al. (1974).

\section{Results}

Effect of the dilution on the amount remaining adsorbed

In these series of experiments two initial phosphate concentrations were used, $3.45 \times 10^{-4}$ and $5.76 \times 10^{-4} \mathrm{M}$, and the conditions were $\mathrm{pH} 5.6$, ionic strength $0.1 \mathrm{M} \mathrm{NaCl}$, and temperature $25^{\circ} \mathrm{C}$ throughout. After three different times of adsorption $(0.25,1$ and $23 \mathrm{~h})$ the 'adsorption suspensions' were diluted from 17 to 3000 times, and samples of the resulting 'desorption suspensions' measured after $0.5 \mathrm{~h}$ to 6 days.

The plots of concentration against time for the 'desorption suspensions' usually showed no significant variation of the concentration after 1-2 days. For example, 


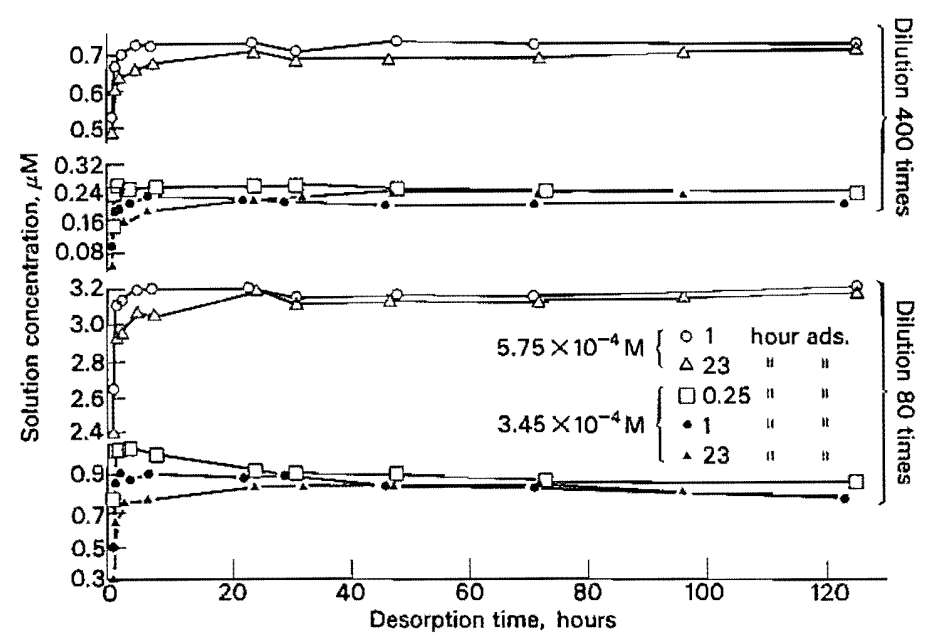

FIG. 1. Variation of the solution concentration with desorption time, 2 dilutions ( 80 and 400 times), after various adsorption times and initial concentrations; ionic strength, $0.1 \mathrm{M} \mathrm{NaCl}$.

Fig. 1 shows the results for two dilutions, 80 and 400 times. Results for the other dilutions are similar and are not shown here. In the first $24 \mathrm{~h}$ the behaviour differed according to which time had been allowed for adsorption. There is a rapid increase in the solution concentration, which seems to pass through a maximum at small adsorption times and dilutions. After $24 \mathrm{~h}$ there is little or no significant difference for the various adsorption times especially when compared with the concentrations at zero desorption time. These results, comparable with those from other authors (e.g. Munns and Fox, 1976), suggest that such 'readsorption' is the effect of a slow component of the adsorption reaction. For desorption times long compared with the adsorption time there is no effect of the latter on the adsorbed amount.

When the amount remaining adsorbed after 5-6 days of desorption for the various dilutions is plotted against the corresponding solution concentration, a 'desorption isotherm' is obtained. As can be seen in Fig. 2, the results lie within a region narrow enough to be considered a single curve, regardless of the adsorption conditions (initial phosphate concentration and time), although the points are scattered at low concentrations. In this region sampling errors, contamination with micro-organisms etc. are likely to have a large effect on the phosphate concentration, and this, combined with the magnifying effect of the large dilution on the experimental errors of the concentrations when calculating the adsorbed phosphate, could be the reason for the scatter of the points for concentrations below $5 \times 10^{-7} \mathrm{M}$.

\section{Effect of adsorption and desorption conditions}

Since the error in the determination of the amount adsorbed seems to increase markedly for dilutions of 800 times or greater, a standard dilution of 700 times, just below this limit, was chosen in order to investigate the influence of various conditions of adsorption and desorption on the amount desorbed.

Adsorption suspensions were made up at 3 different pH values $(4,7,10)$ and 3 different initial phosphate concentrations $\left(4.61 \times 10^{-4}, 6.91 \times 10^{-4}\right.$ and $9.22 \times$ $10^{-4} \mathrm{M}$ ). After 3 adsorption times $(0.25,1$ and $24 \mathrm{~h})$ they were diluted 700 times 


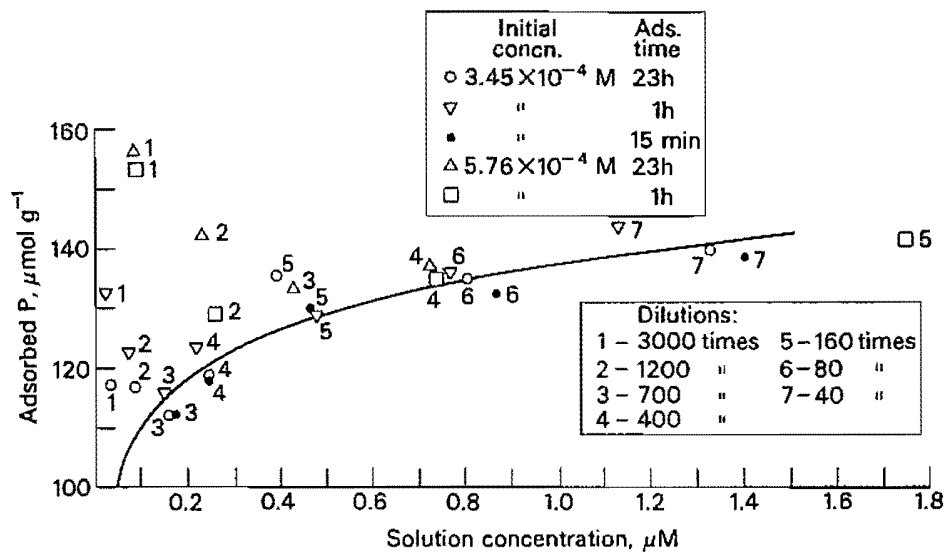

FIG. 2. Variation of the phosphate adsorbed after 5-6 days of desorption with solution concentration, for various dilutions and adsorption conditions; $\mathrm{pH} 5.6$; ionic strength $0.1 \mathrm{M} \mathrm{NaCl}$. Full line: theoretical isotherm at $\mathrm{pH} 5.6$ calculated using the procedures of Bowden $e t$ al. $(1973,1974,1977)$ and the following parameters of the model (Bowden et al., 1977), pzc = 8.0; maximum number of adsorption sites $\left(N_{T}\right)=3 \times 10^{-10}$ moles $/ \mathrm{cm}^{2}$. Electrical capacitance $(G)=2 \times 10^{-12}$ equiv. $\mathrm{cm}^{-2}$ mvolt ${ }^{-1}$. Binding constants; $K_{H_{2} P O_{4}^{-}}=0, K_{H P O_{4}^{2-}}=10^{4}$ Imole ${ }^{-1}, K_{P O_{4}^{3-}}=10^{4} 1 \mathrm{~mole}^{-1}$. These parameters were obtained at high phosphate concentrations where time effects would be small. The Nernst equation was used to calculate the surface potential $\left(\psi_{s}\right)$ since it has been shown to be a good approximation when the surface coverage is not too large (Bowden et al., 1977).

under various desorption conditions of $\mathrm{pH}$, temperature and ionic strength. In some of these experiments $\mathrm{LiCl}$ was used instead of $\mathrm{NaCl}$ as electrolyte.

For a given set of desorption conditions and for a given initial phosphate concentration, a group of graphs was obtained as in Fig. 3. After 1-2 days of desorption, the adsorbed phosphate seems to reach an 'equilibrium' value, unique

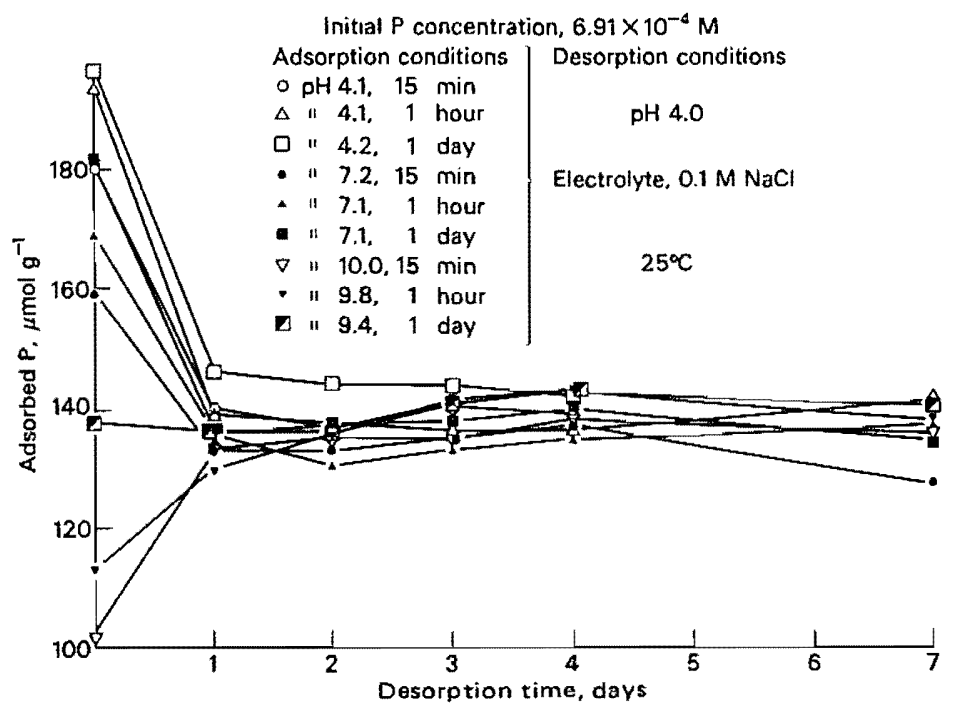

FIG. 3. Examples of the variation of the adsorbed phosphate with desorption time, for various adsorption conditions. 


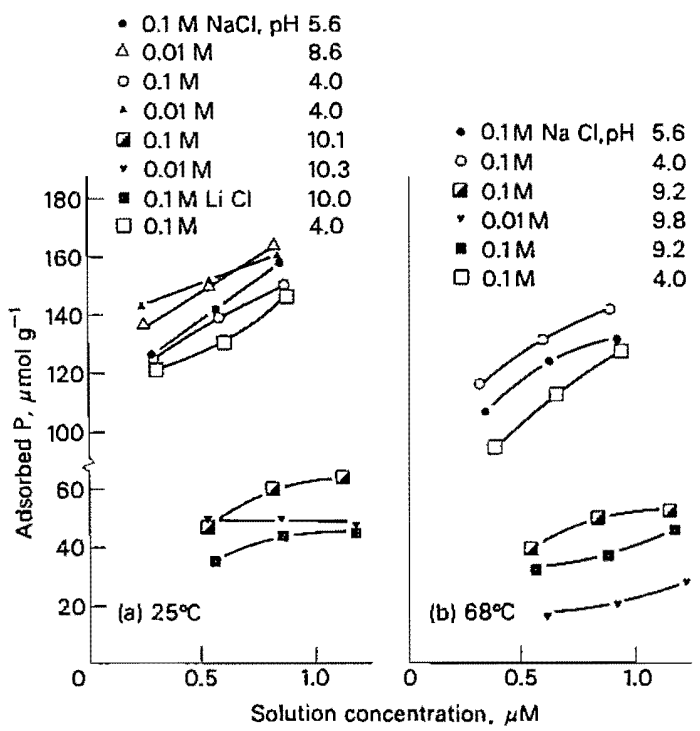

FIG. 4. Plot of average adsorbed phosphate against average solution concentration after 5-6 days of desorption, various desorption conditions: a) $25^{\circ} \mathrm{C}$; b) $68^{\circ} \mathrm{C}$.

for each of these groups, which does not depend upon the time or the $\mathrm{pH}$ of adsorption. Those suspensions with less adsorbed phosphate (at desorption time $=0$ ) than this unique value adsorb phosphate during the first day after dilution, and those with more phosphate adsorbed than the 'equilibrium' value desorb part of it. The average 'equilibrium' values after 5-6 days of the amount of phosphate remaining on the oxide are plotted in Fig. 4 against the corresponding average solution concentrations for various desorption conditions.

Adsorption and desorption at very short reaction times

Some adsorption suspensions were diluted after short adsorption times (1-5 $\mathrm{min}$ ), and samples of the corresponding desorption suspensions were also taken at short times, from $1 \mathrm{~min}$ up to $1 \mathrm{~h}$ with a final sample after 1 day. Fig. 5 shows the results for experiments at $68^{\circ} \mathrm{C}$. The experiments at $25^{\circ} \mathrm{C}$ gave a similar pattern and are not shown here.

The behaviour was similar to the one described in the preceding section: the samples either desorbed phosphate or adsorbed more, depending upon whether they had more or less phosphate adsorbed at desorption time $=0$ than the 'equilibrium' adsorption. The approach to this 'equilibrium' adsorption seemed to be similar to the experiment in Fig. 1 for $15 \mathrm{~min}$ of adsorption and large dilution: there was a very rapid desorption within the first few minutes, slowing down later, so that the variation between 1 and $24 \mathrm{~h}$ was small in most cases. The maximum in the desorption reported for some results in Fig. 1 was not found here, probably because the slow readsorption was reduced by the 700 fold dilution used.

\section{Desorption by 'steps'}

The purpose of this experiment was to establish whether the desorption resulting from an increase in $\mathrm{pH}$ was comparable in speed to that resulting from dilution at 


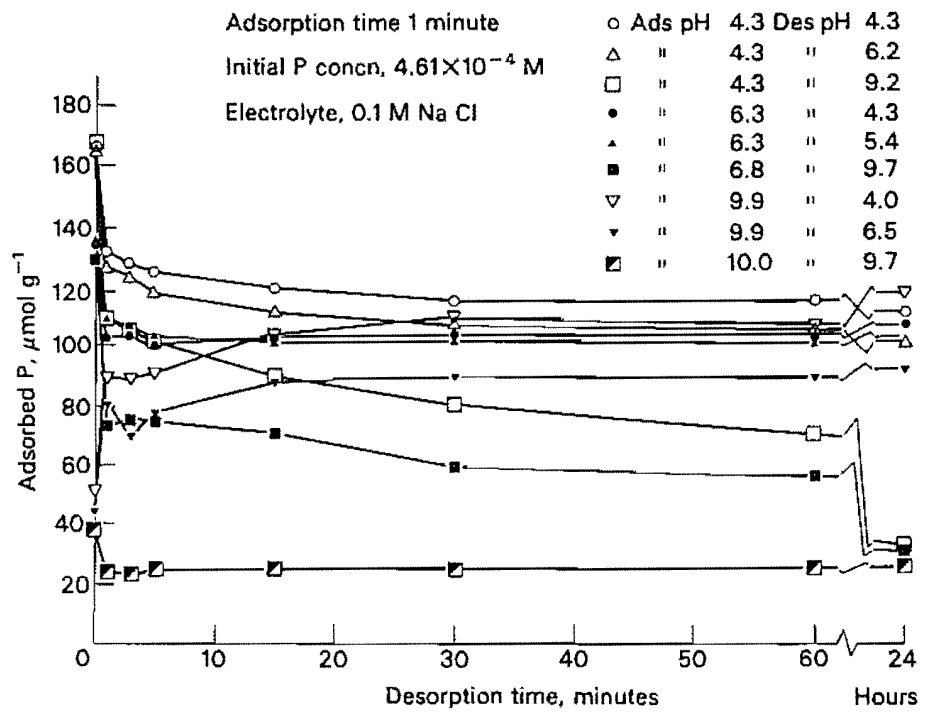

FIG. 5. Examples of the variation of the adsorbed phosphate with desorption time, short-term experiments.

constant $\mathrm{pH}$. In these experiments, the $\mathrm{pH}$ of the desorption suspension was not constant throughout the whole desorption time, but was increased every few days, and samples were taken $1 \mathrm{~h}, 6 \mathrm{~h}, 1$ day and 2-3 days after each change in $\mathrm{pH}$ (Fig. 6, means of eight experiments). The initial phosphate concentration was $4.61 \times 10^{-4} \mathrm{M}$ the adsorption $\mathrm{pH}$ and the initial desorption $\mathrm{pH}$ were 4.0 , and the electrolyte was $0.1 \mathrm{M} \mathrm{NaCl}$.

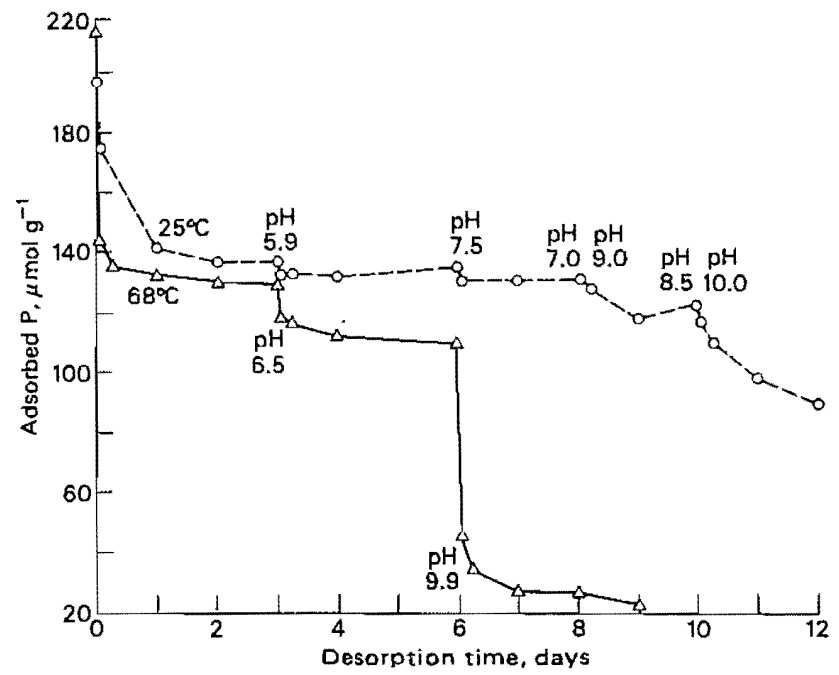

FIG. 6. Variation of the adsorbed phosphate with desorption time following changes in $\mathrm{pH}$; ionic strength $0.1 \mathrm{M} \mathrm{NaCl}$. 
It can be seen that after each pH change the amount adsorbed decreased sharply within the first hour, and later, as happened in the experiments described in previous sections, the rate of desorption decreased markedly. The sharp decrease in the amount adsorbed after each pH change is particularly noticeable for the experiments at $68^{\circ} \mathrm{C}$.

In some cases, probably due to contamination with $\mathrm{CO}_{2}$, the $\mathrm{pH}$ decreased again after some time, and in such cases the amount of phosphate adsorbed increased accordingly.

\section{Experiments at very low concen trations}

If a slow component of the adsorption process is responsible for the difference between adsorption and desorption isotherms, it would be likely to produce small variations in the 'equilibrium' concentrations which would be more noticeable at very low concentrations. For that reason and to minimise experimental errors due to dilution noted for the results reported in Fig. 1, some experiments were made with low initial solution concentrations $\left(1.84 \times 10^{-4}\right.$ and $\left.2.30 \times 10^{-4} \mathrm{M}\right)$ to give equilibrium concentrations equal to or less than $10^{-7} \mathrm{M}$. Desorption suspensions were prepared from suspensions by diluting 100 and 200 times after 3 days of adsorption. Samples were taken from both adsorption and desorption suspensions at various times up to 20 days.

Preliminary experiments in this series showed that the polythene bottles themselves produced a small decrease in solution concentration of phosphate. The decrease was not detectable for higher concentrations. With polycarbonate containers little change in the concentration of phosphate was observed over 3 days and they were used in this series of experiments instead of polythene bottles.

Fig. 7 shows that the solution concentrations for both adsorption and desorption slowly decrease, so that all points tend to approach the 'theoretical' isotherm. For this reason, comparison of desorption and adsorption points corresponding to similar total periods of reaction, i.e. desorption points for 3 days and adsorption points for 6 days (since the desorption experiments were prepared after 3 days of adsorption) show that the apparent discrepancy between both adsorption and desorption points is much less marked. Fig. 8 summarizes adsorption and desorption results at $\mathrm{pH} 4$ from the various series described above. The desorption points have been chosen so that the total time of reaction (adsorption plus desorption) is the same as for the adsorption points, $24 \mathrm{~h}$, except the desorption point $\mathrm{A}$, which corresponds to $24 \mathrm{~h}$ of desorption after $24 \mathrm{~h}$ of adsorption. The results can again be fitted into a single line if the total reaction time is the same. This conclusion was already reached from Fig. 7 for longer reaction times and very low concentrations, and is consistent with results from other authors (e.g. White and Taylor, 1977).

\section{Effect of the adsorption-desorption technique}

Centrifugation has been widely used for separating solid from solution in the adsorption suspensions before adding the desorbing solution. Since this technique may cause the formation of aggregates which could make the solid surface partly inaccessible and decrease or slow down the subsequent desorption, a series of experiments was carried out to test such hypothesis. The same procedure described by Hingston et al. (1974) was used, and after adding the desorbing solution the oxide was redispersed by different means including ultra-sonic dispersion. If the hypothesis was correct, the more energetic the redispersion the more phosphate would be desorbed. Little or no difference was observed. 


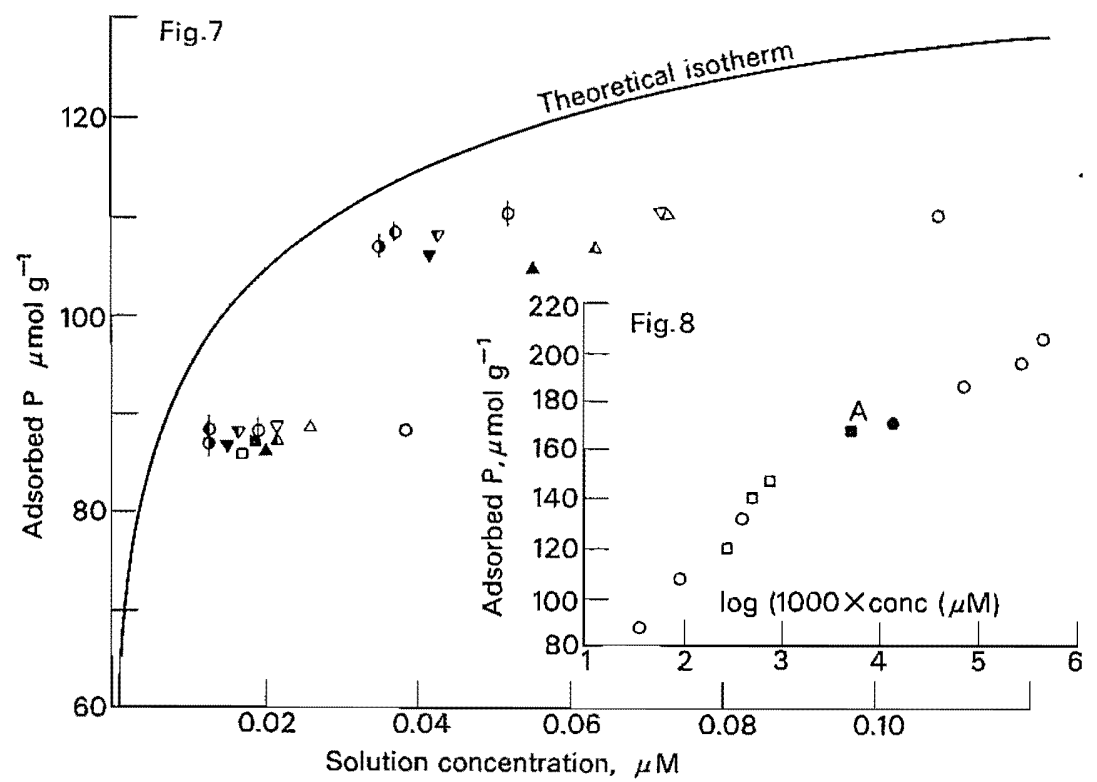

FIG. 7. Position of adsorption and desorption points for various reaction times. Adsorption $\mathrm{pH} 4$; desorption $\mathrm{pH} \mathrm{4;} \mathrm{ionic} \mathrm{strength} 0.1 \mathrm{M} \mathrm{NaCl}$. Theoretical isotherm calculated using parameters given for Figure 2.

$\begin{array}{ll}\text { Adsorption } \\ 0 & 3 \mathrm{~d} \\ \Delta & 6 \mathrm{~d} \\ \nabla & 13 \mathrm{~d} \\ \phi & 19 \mathrm{~d}\end{array}$

Desorption

(A3d. dilution 100. $7 \mathrm{~d} \nabla 10 \mathrm{~d} \phi 16 \mathrm{~d}$

(A 3d. dilution $200.07 \mathrm{~d} \vee 10 \mathrm{~d} \phi 16 \mathrm{~d}$

FIG. 8. (Inset in Fig. 7). Comparison of various adsorption and desorption results at pH 4, ionic strength $0.1 \mathrm{M} \mathrm{NaCl}$.
- Adsorption
$\square$ Desorption
-) Desorption, technique
m of Hingston et al. (1974)

\section{Discussion}

Even though the results obtained here suggest that, given enough time, adsorption is both reversible (Figs. 2, 7, 8) and in accord with the Stern model (Bowden, 1973), extremely high dilutions may be required to desorb completely the phosphate. For example, given a point on the theoretical isotherm of solution concentration $C_{1}$ $\mu \mathrm{mol} . \mathrm{ml}^{-1}$ and adsorbed phosphate $X_{1} \mu \mathrm{mol} . \mathrm{g}^{-1}$ for a solid : solution ratio $A \mathrm{~g} \cdot \mathrm{ml}^{-1}$, the volume of phosphate-free solution that has to be added to $1 \mathrm{ml}$ of the system in order to reach a second point of composition $C_{2}$ and $X_{2}$ is, in $\mathrm{ml}$,

$$
V=\frac{\left(X_{1}-X_{2}\right) A+C_{1}}{C_{2}}-1
$$

For the system used here $C_{1}=3.45 \times 10^{-5} \mathrm{M}, X_{1}=194.9 \mu \mathrm{mol} . \mathrm{g}^{-1}$, and $A=$ $0.0021 \mathrm{~g}$ goethite $\mathrm{ml}^{-1}$. Thus, after a dilution of 100000 times (much higher than 
the maximum dilution of 3000 used here) the solid still retains 50 per cent of the amount adsorbed at the starting point.

The above findings do not exclude the possibility of adsorbing sites being heterogeneous, as results of isotopic exchange have suggested (Atkinson et al., 1972). In fact, the maximum observed in some plots of the solution concentration against desorption time after times of adsorption shorter than 1 day (Fig. 1) suggests the occurrence of more than one process during both the adsorption and desorption reactions, ranging from the very fast to the very slow. The results in Figs. 5 and 6 also demonstrate the existence of a range of desorption rates, while Fig. 9 shows that a range exists for adsorption. These figures show that a large proportion of the reactions are fast. Adsorption is extremely fast and is faster the lower the $\mathrm{pH}$. At $\mathrm{pH} 4.0$ at least 90 per cent of the adsorption at $24 \mathrm{~h}$ is complete after one min and hence is too fast to measure.

Some authors have suggested that the slow process could be related to diffusion of phosphate into the solid (Ryden et al., 1977), or, in the case of soils, into the soil aggregates. This last hypothesis may well be true for experiments with un-

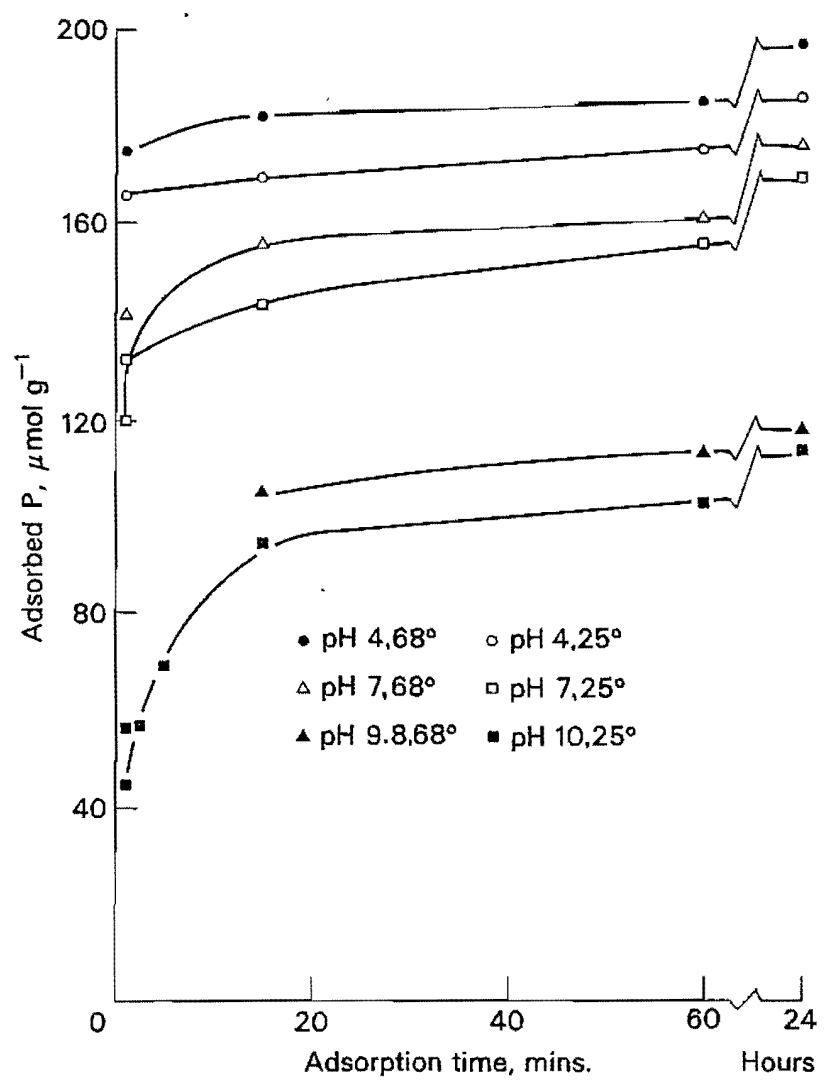

FIG.9. Variation of the adsorbed phosphate with adsorption time for various adsorption conditions; ionic strength $0.1 \mathrm{M} \mathrm{NaCl}$. 
shaken soils, but it is very unlikely in the present experiments, where the goethite is strongly dispersed and is not allowed to settle at any time during the experiments.

Fig. 6 shows that there is a very rapid approach to a new equilibrium each time the $\mathrm{pH}$ is raised, the speed of which is comparable to the adsorption process (Fig. 9). If the desorption in each step was related to some kind of diffusion process out of the lattice, the change in $\mathrm{pH}$ should not increase the rate so sharply. Thus, if there is a diffusion component in the process, its contribution is negligible for times shorter than a few days.

The fact that there is no marked difference between desorption experiments after $1 \mathrm{~min}$ or $15 \mathrm{~min}$ of adsorption (cf. Figs. 1 and 5) suggests that any slow process has little effect for times shorter than $15 \mathrm{~min}$.

Results in Fig. 4 have some features which are worth noting.

I) For a given ionic strength, the amount of phosphate remaining on the oxide decreases as the $\mathrm{pH}$ increases. The exceptions are the curves for $\mathrm{pH} 4$ and 5.6 , which cross each other for ionic strength $0.01 \mathrm{M}$.

II) At $\mathrm{pH}$ values below the pzc e.g. $\mathrm{pH} 4.0$ and $5.6,0.01 \mathrm{M} \mathrm{NaCl}$ leaves more phosphate on the oxide than $0.1 \mathrm{M} \mathrm{NaCl}$, and the opposite is true for pH values above the pzc; e.g. compare pH 10.1 and 10.3 (Fig. 4a) and also pH 9.2 and 9.6 (Fig. 4b).

III) The amount of phosphate remaining on the oxide is greater at $25^{\circ} \mathrm{C}$ than at at $68^{\circ} \mathrm{C}$, with the exception of $\mathrm{LiCl}$ at $\mathrm{pH} 10$, which does not show a significant difference between the two temperatures (compare Figs. $4 \mathrm{a}$ and $\mathrm{b}$ ). Nevertheless, this exception can be due to the small difference in $\mathrm{pH}$ between both series of experiments.

IV) All other conditions being equal, $\mathrm{LiCl}$ leaves less phosphate adsorbed than $\mathrm{NaCl}$ (see Fig. $4 \mathrm{a}$ and $\mathrm{b}$ ).

The features I) and II) are fully compatible with the effect of $\mathrm{pH}$ and ionic strength on the adsorption isotherms calculated by the Stern model. The greater desorption with increasing $\mathrm{pH}$ results from an increase in competition from hydroxyl ions, and the decrease in positive charge or increase in negative charge on the surface resulting in a lessening of the attraction or a repulsion, respectively, between the surface and the anion. This effect is further amplified if the anion increases its negative charge as the $\mathrm{pH}$ is increased.

At $\mathrm{pH}$ values below the pzc, increasing the ionic strength depresses the positive diffuse double layer potential and hence reduces the attraction between the anion and the surface, hence adsorption is decreased. Above the pzc raising the ionic strength decreases the negative diffuse double layer potential which reduces the repulsion between the negative surface and the anion, hence adsorption is increased.

\section{REFERENCES}

ATKINSON, R. J., POSNER, A. M. and QUIRK, J. P. 1972. Kinetics of heterogeneous isotopic exchange reactions. Exchange of phosphate at the $\alpha-\mathrm{FeOOH}$-aqueous solution interface. J. inorg. nucl. Chem. 34, 2201-11.

BARROW, N. J. and SHAW, T. C. 1975. The slow reactions between soil and anions: 5. Effects of period or prior contact on the desorption of phosphate from soils. Soil Sci. 119, 311-20.

BOWDEN, J. W. 1973. Models for ion adsorption on mineral surfaces. Ph.D Thesis, Univ. Western Australia.

HINGSTON, F. J., POSNER, A.M. and QUIRK, J. P. 1972. Anion adsorption by goethite and gibbsite. I. The role of the proton in determining adsorption envelopes. J. Soil Sci. 23, 177-92.

1974. Anion adsorption by goethite and gibbsite. II. Desorption of anions from hydrous oxide surfaces. Ibid $25,16-26$. 
KAFKAFI, U., POSNER, A. M. and QUIRK, J. P. 1967. Desorption of phosphate from kaolinite. Proc. Soil Sci. Soc. Am. 31, 348-53.

MULJADI, D., POSNER, A. M. and QUIRK, J. P. 1966. The mechanism of phosphate adsorption by kaolinite, gibbsite and pseudoboehmite. J. Soil Sci. 17, 212-47.

MUNNS, D. N. and FOX, R. L. 1976. The slow reaction which continues after phosphate adsorption: kinetics and equilibrium in some tropical soils. Proc. Soil Sci. Soc. Am. 40, $46-51$.

NEOH, L. S. 1975. Desorption of phosphate from goethite. Ph.D. Thesis, Univ. Westem Australia. RYDEN, J.C. and SYERS, J.K. 1977 . Desorption and isotopic exchange relationships of phosphate sorbed by soils and hydrous ferric oxide gels. J. Soil Sci. 28, 596-609.

- MCLAUGHLIN, J, R. and SYERS, J. K. 1977. Time-dependent sorption of phosphate by soils and hydrous ferric oxides. Ibid. 28, 585-95.

WHITE, R. E., and TAYLOR, A. W. 1977. Reactions of soluble phosphate with acid soils: the interpretation of adsorption-desorption isotherms. Ibid. 28, 314-28.

(Received 7 October 1978) 